\title{
Une percée décisive dans la génétique épidémiologique de la maladie d'Alzheimer : rôle de l'apolipoprotéine $E$
}

Malgré de multiples efforts, génétique et épidémiologie de la maladie d'Alzheimer sont encore mal connues. On a bien identifié, dans des formes familiales, un locus sur le chromosome 21 , codant pour le précurseur du peptide $\beta$-amyloïde ou APP, et un locus, dont le gène n'est pas identifié, sur le chromosome $14\left(\mathrm{~m} / \mathrm{s} n^{\circ} 10\right.$, vol. 8, p. 1115). Mais les cas dont ils sont responsables sont très minoritaires. Un troisième locus, situé sur le chromosome 19, n'avait pas fait sa preuve [1]. Il apparaît aujourd'hui que ce dernier pourrait être de loin le plus important, après les révélations remarquables du laboratoire de A. Roses à Duke University, Durham, (NC, USA). Ces chercheurs ont en eff et découvert, non plus dans de rares familles, mais dans plusieurs dizaines, et aussi dans de nombreuses formes sporadiques, beaucoup plus fréquentes que les formes familiales, une liaison avec un allèle du gène de l'apolipoprotéine $\mathrm{E}$ (ApoE), situé sur le chromosome 19 en 19q13.2. Trois allèles se partagent la population, ApoE 2, 3, 4, qui ne different entre eux que par un ou deux acides aminés. Dans la population générale, c'est ApoE 3 qui prédomine : $90 \%$ des individus en possèdent une copie, plus de $60 \%$ en ont deux ; à l'opposé, $31 \%$ ont une copie de ApoE 4, et $10 \%$ en ont deux. La première observation [2] fut de s'apercevoir que chez les malades la forme 4 devenait la plus fréquente. Le groupe fut ainsi incité à entreprendre une étude plus ambitieuse : elle porta [3] sur 42 familles à début tardif (aucune atteinte avant 60 ans). La proportion de sujets affectés passait de $20 \%$ quand seuls étaient présents les allèles 2 et 3 à $47 \%$ en pré- sence d'un allèle 4 , et à $91 \%$ pour deux allèles 4 , comme le montre le Tableau I. La probabilité était hautement significative, et le risque augmentait d'un facteur de presque 3 pour chaque allèle ApoE 4, de sorte que les risques d'un individu à génotype $4 / 4$ sont 8 fois plus élevés d'être atteint que pour les sujets $2 / 3$ ou $3 / 3$. Avec le génotype $4 / 4$, pratiquement tous les sujets membres de ces familles sont atteints. Les auteurs font toutefois remarquer que 19 des 95 sujets malades examinés dans cette étude, ainsi que 64 des 176 cas sporadiques vérifiés par autopsie décrits dans un travail actuellement sous presse (in [3]) ne portent pas l'allèle 4. Sur les 42 familles de l'étude précitée, 12 étaient dans ce cas, ce qui suggère l'existence d'autres sources de risque génétique. Peut-on, à l'hcure actuelle, évaluer l'importance de ces découvertes? Sur le plan épidémiologique, elle est certainement considérable. C'est la première fois que l'on trouve une rela- tion génétique valable pour unc majorité des formes familiales et un grand nombre de formes sporadiques. Sur le plan de la physiopathologie, la réponse est moins claire. Il reste possible qu'il s'agisse seulement d'un marqueur, et que le gène responsable soit encore à découvrir. Mais des arguments existent en faveur d'un rôle direct de l'ApoE. Cette protéine, un transporteur du cholestérol dans le sang, est, dans le système nerveux central, produite et sécrétée par les astrocytes. Pourquoi la faible différence entre les trois allèles a-t-elle une telle influence sur la susceptibilité à l'Alzheimer? Les études anatomopathologiques ont montré que, dans le cerveau des malades, on trouvait par des méthodes immunologiques de l'A poE liée aux trois lésions caractéristiques: les plaques séniles, les dépôts d'amyloïde vasculaire, les filaments neurofibrillaires. Et il semble bien que l'ApoE 4 se lie plus vite et plus solidement au peptide $\beta$ amyloïde que le type ApoE 3, plus

\begin{tabular}{|c|c|c|c|c|}
\hline \multicolumn{5}{|c|}{$\begin{array}{l}\text { POURCENTAGE DE SUJETS AFFECTÉS } \\
\text { POUR CHAQUE GÉNOTYPE D'ApoE. (D'après [3]). }\end{array}$} \\
\hline ApoE génotype & Hon & mes & Femmes & Combinés \\
\hline $2 / 2$ & - & (0) & - & (0) \\
\hline $2 / 3$ & 28,6 & (7) & $11,1 \quad$ (9) & 18,8 \\
\hline $3 / 3$ & 7,1 & (28) & $28,6 \quad(49)$ & 20,8 \\
\hline $2 / 4$ & 50,0 & (2) & 0,0 & 20,0 \\
\hline $3 / 4$ & 38,3 & (47) & 54,5 & 47,8 (113) \\
\hline $4 / 4$ & 91,7 & (12) & $90,9 \quad(11)$ & $91,3 \quad(23)$ \\
\hline Total & 35,4 & (96) & 44,2 (138) & $40,6(234)$ \\
\hline
\end{tabular}

Le nombre de sujets pour chaque groupe est entre parenthèses. 
commun. Au total, si l'on admet que le dépôt du peptide amyloïde est la lésion cruciale de la maladie d'Alzheimer, l'A poE pourrait agir comme une molécule " chaperonne" pathologique rendant insoluble le peptide et favorisant la production des lésions [4].

Les résultats que nous venons de relater soulèvent des problèmes médicaux d'une extrême importance. Ils doivent d'abord être confirmés, et de multiples études s'y emploient, lancées dans des ethnies différentes. S'ils le sont, ils introduiront, notamment dans les formes familiales, une notion de risque énorme pour les porteurs d'un, et surtout de deux allèles d'ApoE 4. Tant que l'on ne dispose pas de moyens thérapeutiques pour retarder ou empêcher chez les sujets à risque la survenue de la maladie, le seul résultat que l'on obtiendra sera de crécr un sentiment d'anxiété chez les futurs malades, bien avant l'échéance fatidique. D'après le commentaire de J. Travis dans Science [4], le jugement de Salomon nécessaire pour choisir la meilleure attitude n'a pas encore été rendu.

Une dernière remarque : la région du chromosome 19 qui porte $\mathrm{ApoE}$ est particulièrement riche en gènes pathologiques importants. On y trouve notamment celui de la dystrophie myotonique.

J.C.D.

1. Pericak-Vance MA, Bebout JL, Gaskell PC, el al. Linkage studies in familial Alzheimer disease : evidence for chromosome 19 linkage. Am J Hum Genet 1991; 48 : 1034-50.

2. Strittmatter WJ, Saunders AM, Sehmechel D, Pericak-Vance MA, Enghild D, Salvesen VS, Roses AD. Apolipoprotein E : high-avidity binding to $\beta$ amyloid and increased frequency of type 4 allele in late-onset familial Alzheimer disease. Proc Natl Acad Sci USA 1993 ; 90 ; 1977-81.

3. Corder EH, Saunders AM, Strittmatter WJ, Schmechel DE, Gaskell PC, Small GW, Roses AD, Haines JL, Pericak-Vance MA. Gene dose of apolipoprotein $\mathrm{E}$ type 4 allele and the risk of Alzheimer's disease in late onset families. Science 1993 ; 261: 921-3.

4. Travis J. New piece in Alzheimer's puzzle. Science 1993 ; 261 : 828-9.

$\mathrm{m} / \mathrm{s} n^{\circ} 10 \mathrm{vol} .9$, octobre 93
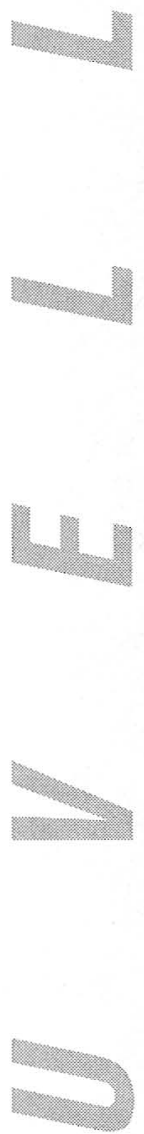